\title{
ASSISTÊNCIA DE ENFERMAGEM A DOENÇA ARTERIAL OBSTRUTIVA PERIFÉRICA: UM RELATO DE EXPERIÊNCIA
}

\section{NURSING CARE FOR PERIPHERAL ARTERIAL DISEASE: AN EXPERIENCE REPORT}

\section{CUIDADOS DE ENFERMERÍA PARA LA ENFERMEDAD ARTERIAL PERIFÉRICA: UM REATO DE EXPERIENCIA}

\author{
Tamires Gomes dos Santos ${ }^{1,}$ Fernanda Silva Santos ${ }^{2,}$ Márcia Tasso Dal Poggetto ${ }^{3,}$ Fernanda \\ Bonato Zuffi ${ }^{4}$
}

\section{RESUMO}

Trata-se de um relato de experiência no cuidado a uma paciente com o diagnóstico médico de doença arterial obstrutiva periférica acompanhada pelo Ambulatório de Feridas, projeto desenvolvido por uma Residência Integrada Multiprofissional em Saúde do interior de Minas Gerais. A paciente necessitou de acompanhamento por apresentar gangrena em pododáctilo $1^{\circ}, 2^{\circ}$ e $3^{\circ}$ e úlcera em dorso do pé direito. Foram traçados os diagnósticos de enfermagem, para que fossem estabelecidas as devidas intervenções, sendo os diagnósticos: perfusão tissular periférica ineficaz, mobilidade física prejudicada, risco de infecção e dor aguda. Contudo, evidenciou-se a importância da Sistematização da Assistência de Enfermagem, uma ferramenta essencial para o desempenho de intervenções e de gerenciamento desse cuidado favorecendo a atuação do enfermeiro em uma abordagem integral além de fomentar outras investigações.

Descritores: Doença Arterial Periférica. Cuidados de Enfermagem. Estudos de Casos.

\begin{abstract}
It is an account of experience in the care of a patient with a medical diagnosis of peripheral arterial disease accompanied by the Wound Clinic, a project developed by a Multidisciplinary Integrated Residence in Health of Minas Gerais. The patient required follow-up to present toe gangrene in 1st, 2nd and 3rd and ulcers on the dorsum of the right foot. Nursing diagnoses were drawn, so that appropriate interventions were established, and the Diagnostics: Peripheral tissue perfusion ineffective, impaired physical mobility, risk of infection and acute pain. However, it highlighted the importance of systematization of nursing care, an essential tool for the performance of interventions and management of this care favoring the nurse's performance in a holistic approach as well as foster further investigations. Descriptors: Peripheral Arterial Disease. Nursing Care. Case Studies.
\end{abstract}

\section{RESUMEN}

Es un relato de experiencia en el cuidado de un paciente con un diagnóstico médico de la enfermedad arterial periférica acompañada por la Clínica de Heridas, un proyecto desarrollado por un integrado Residencia Multidisciplinaria de Salud de Minas Gerais. El paciente requiere seguimiento de la actualidad gangrena dedo del pie en primera, segunda y tercera y las úlceras en el dorso del pie derecho. Diagnósticos de enfermería fueron extraídas, de modo que se establecieron las intervenciones apropiadas y de los diagnósticos: la perfusión

1 Enfermeira. Residente multiprofissional em Saúde do Idoso da UFTM. E-mail: tamiresgomes_santos@hotmail.com.

2 Enfermeira. Mestre em Atenção à Saúde da UFTM. E-mail: nandasantosfmtm@yahoo.com.br;

3 Enfermeira. Mestre em Enfermagem. Professora Assistente do Departamento de Enfermagem na Assistência Hospitalar do CGE/UFTM. E-mail: marciatasso60@yahoo.com.br;

4 Enfermeira. Mestre em Enfermagem. Docente do Curso de Graduação em Enfermagem da UFTM. E-mail: fbzuffi@yahoo.com.br; 
tisular periférica ineficaz, la movilidad física alterada, riesgo de infección y dolor agudo. Sin embargo, se destacó la importancia de la sistematización de la asistencia de enfermería, una herramienta esencial para la realización de las intervenciones y la gestión de este tipo de atención que favorece la actuación de la enfermera en un enfoque holístico, así como fomentar nuevas investigaciones.

Descriptores: Enfermedad Arterial Periférica. Atención de Enfermería. Estudios de Casos.

\section{INTRODUÇÃO}

A Doença Arterial Obstrutiva Periférica (DAOP) é um conjunto de condições crônicas ou agudas decorrentes da obstrução arterial, a qual gera diminuição do fluxo de sangue para as extremidades, principalmente as inferiores ${ }^{1}$. Essa doença em membros inferiores pode gerar amputações e também risco de desenvolvimento de doenças cardio e cerebrovasculares $\operatorname{associadas}^{2}$.

Dentre os principais fatores de risco para o seu desenvolvimento estão: Diabetes Mellitus (DM), Hipertensão Arterial Sistêmica (HAS), hipertrigliceridemia e tabagismo ${ }^{3}$. O DM é caracterizado pelo excesso de glicemia, resultante de uma resistência à ação da insulina e/ou um defeito na secreção da mesma ${ }^{4}$. Seu desenvolvimento ocorre devido à incapacidade da célula beta em responder à crescente demanda de insulina para compensar a resistência à mesma ${ }^{5}$.

Por ser uma doença multissistêmica, o DM é capaz de ocasionar diversas complicações nos indivíduos que o possuem. Essas complicações podem ser classificadas em microvasculares, como a neuropatia, a nefropatia e a retinopatia; e macrovasculares, como o acidente vascular encefálico, o infarto agudo do miocárdio e a doença vascular periférica ${ }^{4}$.

Ainda, evidencia-se um risco maior para amputação em diabéticos com DAOP, sendo importante diagnosticar precocemente o DM para prevenir a incapacidade e perda de membro ${ }^{2}$. Observa-se que a amputação está vinculada a diversos sentimentos, como a tristeza pela perda de uma parte do corpo, a apreensão pela possível dependência nas atividades diárias, bem como alívio da dor e o desconforto pelo odor causados pela lesão ${ }^{6}$.

Outro fator de risco é a HAS, doença multifatorial caracterizada por níveis elevados da pressão arterial, associado frequentemente com alterações na funcionalidade e/ou na estrutura de órgãos alvos como: coração, encéfalo, rins e vasos sanguíneos. Assim, observa-se que dentre as condições clínicas associadas a HAS está a DAOP ${ }^{7}$. Corroborando com essa associação, estudos realizados com indivíduos com DAOP verificou que $71 \%$ apresentavam $\mathrm{HAS}^{8}$. 
Assim como a DM e a HAS já mencionadas, a dislipidemia também tem sido evidenciada como fator predisponente para a $\mathrm{DAOP}^{8}$. A dislipidemia é a caracterizada pelo distúrbio que altera os níveis séricos de lipídeos ${ }^{9}$. Estudo realizado com pessoas com DAOP verificou que $89 \%$ dos mesmos apresentavam dislipidemia ${ }^{8}$.

Somando-se aos fatores acima citados, a American Diabetes Association (ADA), destaca que o tabagismo associado ao diabetes, representa um dos mais importantes fatores de risco para $\mathrm{DAOP}^{2}$. Contudo, por serem fatores modificáveis, devem ser analisados com o intuito de orientar e intervir, com intuito de diminuir custos para o sistema de saúde ${ }^{3}$.

A DAOP também foi evidenciada em outro estudo que verificou os impactos na qualidade de vidas dos indivíduos, sendo afetados os quatro domínios de qualidade de vida, com maior destaque para o domínio físico, seguido, em ordem decrescente, pelo ambiental, psicológico e social $^{10}$. Contudo, tendo em vista que essa doença ocasiona diversas complicações e afeta diferentes aspectos da qualidade de vida dos indivíduos que a possuem, faz-se necessário estudos voltado a assistência à saúde adequada para essa doença. Nesse sentido, destaca-se o papel do enfermeiro como prestador de uma assistência à saúde qualificada.
Assim, este estudo adveio da experiência da principal autora como enfermeira da residência multiprofissional em saúde do idoso na sua prática assistencial em um ambulatório de feridas criado por meio de um projeto de extensão. Objetivou-se relatar o caso a partir da assistência prestada a uma usuária diabética com DAOP, bem como demonstrar diagnósticos de enfermagem e as intervenções elencada para esse caso.

\section{MÉTODO}

Trata-se de um estudo descritivo, do tipo relato de experiência, realizado durante prestação de assistência de enfermagem em um ambulatório de feridas, projeto de extensão da residência multiprofissional, de uma cidade do interior de Minas Gerais no período de março de 2014 a fevereiro de 2015. O projeto de extensão encontra-se registrado na PROEXT UFTM, com protocolo $\mathrm{n}^{\circ}$ 217.114, da Universidade Federal do Triângulo Mineiro.

Esse método foi escolhido por se tratar de uma ferramenta descritiva, que gera uma reflexão sobre ações que estiveram presentes em uma situação vivenciada no âmbito profissional de interesse para a academia científica ${ }^{11}$. As realizações de relatos de experiências, mesmo em casos isolados, contribuem significativamente para construir 
conhecimentos $^{12}$. Esse relato, em específico, abordou questões da sistematização de enfermagem a uma pessoa com lesões cutâneas advindas do DAOP, englobando todas as repercussões sobre o seu estado de saúde.

\section{APRESENTAÇÃO DO CASO}

O relato trata-se da assistência prestada a uma usuária do Sistema Único de Saúde, entre novembro de 2014 a fevereiro de 2015. A paciente foi representada pelas iniciais M.A.D, 58 anos, sexo feminino, viúva, pensionista, morando em casa própria com a filha, alfabetizada, hipertensa, diabética e sedentária.

O acompanhamento ambulatorial ocorreu semanalmente, com a realização de curativos em $1^{\circ}, 2^{\circ}$ e $3^{\circ}$ pododáctilos e em dorso do pé direito. Para desenvolvimento deste relato de experiência, além dos cuidados prestados, foi realizada a análise do prontuário. $\mathrm{O}$ registro da assistência foi descrito nas folhas de procedimento e registrado em um caderno de controle.

Em outubro de 2014, a paciente foi internada em uma unidade de Clínica Médica de um hospital Universitário na região do Triângulo Mineiro, apresentando-se com os seguintes diagnósticos médicos: Diabetes Mellitus tipo 2 há 24 anos, Hipertensão Arterial
Sistêmica há 8 anos, hipotireoidismo, cardiopatia chagásica, dislipidemia e doença arterial obstrutiva periférica com gangrena em pododáctilo $1^{\circ}$ e $3^{\circ}$ e úlcera em dorso do pé direito. Foi realizada uma arteriografia no membro inferior acometido, que evidenciou: poplítea com estenose de $90 \%$ distal, tibial anterior com estenose de $80 \%$ proximal, fibular com estenose de $90 \%$ proximal e tibial posterior ocluída.

Inicialmente, a equipe médica responsável decidiu que não era indicado tratamento cirúrgico, pela impossibilidade de realização da angioplastia devido à localização das lesões, agravamentos e maiores complicações passíveis de ocorrer com o procedimento. A conduta seguida pela equipe médica foi aguardar a completa mumificação dos pododáctilos para posterior amputação espontânea. Nesse período de internação, foi solicitada a avaliação da enfermeira residente no momento da alta hospitalar, e a mesma realizou o encaminhamento da paciente para o ambulatório de feridas no qual fazia atendimento semanal.

No ambulatório, além dos curativos nos pododáctilos e no dorso do pé direito, foram feitas orientações sobre como fazer o curativo no domicilio e os cuidados necessários. Durante o acompanhamento, observou-se que o membro inferior direito estava edemaciado $(+2 /+4)$, dificuldade em 
deambular, com necessidade do auxílio da cuidadora e a paciente relatava presença de dor constante no local. $\mathrm{O} 2^{\circ}$ pododáctilo também iniciou um processo de gangrena devido a aproximação com os outros. Com a realização dos curativos, houve cicatrização da úlcera em dorso do membro. No mês de fevereiro de 2015, os pododáctilos acometidos por gangrena foram amputados.

\section{DISCUSSÃO}

Em sua prática assistencial, os enfermeiros apresentam um trabalho gerencial e assistencial de forma interligada. A Sistematização da Assistência de Enfermagem (SAE), é uma ferramenta essencial para o desempenho de intervenções e de gerenciamento desse cuidado $^{13}$.

No Brasil, a classificação utilizada é

a North American Nursing Diagnosis

Association (NANDA) que traz o termo diagnóstico de enfermagem como "um julgamento clínico sobre a resposta de um indivíduo, uma família ou uma comunidade com relação a problemas de saúde reais ou potenciais/ processos de vida que fornecem a base para uma terapia definitiva que busca alcançar resultados nos quais a enfermagem é necessária"14 ${ }^{\text {. A }}$ utilização dessa classificação possibilita a constatação das ações de enfermagem dando, assim visibilidade à profissão, conferindo cientificidade ${ }^{15}$.

Diante da situação exposta, dentre os inúmeros diagnósticos de enfermagem possíveis, foram (listados) - de acordo com a taxonomia NANDA e intervenções de enfermagem ${ }^{16}$.

A seguir serão apresentados no quadro os diagnósticos de enfermagem identificados e as intervenções de enfermagem:

Quadro I: Diagnósticos e Intervenções de Enfermagem

\begin{tabular}{|c|c|}
\hline DIAGNÓSTICO DE ENFERMAGEM & INTERVENÇÕES \\
\hline $\begin{array}{l}\text { Perfusão tissular periférica ineficaz, relacionada a diabetes } \\
\text { mellitus, hipertensão e estilo de vida sedentário, } \\
\text { evidenciada por edema, cicatrização de ferida periférica } \\
\text { retardada e dor em extremidade. }\end{array}$ & $\begin{array}{l}\text { - Realizar controle de líquidos; } \\
\text { - Cuidados com os pés. } \\
\text { - Monitorização de sinais vitais. } \\
\text { - Supervisão da pele para verificar ruptura tissular e } \\
\text { prevenir úlceras arteriais. } \\
\text { - Orientar sobre alimentação saudável. }\end{array}$ \\
\hline $\begin{array}{l}\text { Mobilidade física prejudicada, relacionada a dor e } \\
\text { desconforto, evidenciada por instabilidade postural, } \\
\text { movimentos lentos e mudanças na marcha }\end{array}$ & $\begin{array}{l}\text { - Encorajar a promoção de exercícios para o equilíbrio, } \\
\text { alongamento e controle muscular. } \\
\text { - Realizar o controle do ambiente. } \\
\text { - Incentivar o autocuidado. } \\
\text { - Cuidados com os pés. } \\
\text { - Realizar a supervisão da pele para prevenção de } \\
\text { lesões. }\end{array}$ \\
\hline $\begin{array}{l}\text { Risco de infecção relacionado a defesas prima } \\
\text { inadequadas (tecido traumatizado) } \mathrm{e} \text { doença crô }\end{array}$ & rar sinais e sintomas sistêmicos e locais de \\
\hline
\end{tabular}




\begin{tabular}{|l|l|}
\hline (hipertensão arterial e diabetes mellitus). & $\begin{array}{l}\text { Examinar a pele e membranas mucosas na busca de } \\
\text { hiperemia, calor exagerado ou drenagem. } \\
\text { Promover ingesta nutricional adequada. } \\
\text { Pninar ao paciente e familiares a forma de evitar } \\
\text { infecções. } \\
\text { - Manter assepsia para o paciente de risco. }\end{array}$ \\
\hline $\begin{array}{l}\text { Dor aguda relacionada a agentes lesivos e evidenciada por } \\
\text { relato verbal e evidência observada de dor e } \\
\text { comportamento de proteção. }\end{array}$ & $\begin{array}{l}\text { Administrar medicação prescrita para a dor, antes da } \\
\text { realização do curativo. } \\
\text { Identificar com o paciente fatores de melhora e piora } \\
\text { da dor. } \\
\text { Avaliar dor: local, características, início/duração, } \\
\text { frequência, intensidade ou gravidade da dor e fatores } \\
\text { precipitantes. } \\
\text { Realizar monitorização dos sinais vitais. }\end{array}$ \\
\hline
\end{tabular}

formando uma atenção integral e de

Para a realização dos curativos, inicialmente a ferida do dorso do pé era limpa com solução fisiológica aquecida; nos pontos de necrose aplicava-se papaína $6 \%$, já que essa tem sido apontada pelos seus efeitos desbridante, antiinflamatório e bactericida, que acarretam a formação de tecido de granulação ${ }^{17}$. Já nas áreas de gangrena dos pododáctilos, foi prescrito pelo médico uso de apenas digliconato de clorexidina solução alcoólica $0,5 \%$ para fazer antissepsia.

\section{CONCLUSÕES}

Assim, verifica-se que DAOP exige uma maior atenção e sensibilidade do profissional de saúde, visto que o paciente que convive com doenças crônicas não transmissíveis apresenta diversas limitações em seu estilo de vida e interação com o ambiente necessitando de cuidados específicos. Sendo necessário um cuidado sistematizado da enfermagem, que não deve ser feita de forma empírica, mas pautada em evidências e cientificidade, qualidade para o paciente.

O uso dos diagnósticos nessa prática assistencial forneceu desafios constantes e impulsionou o pensamento crítico para a elaboração das atividades, fundamentadas na literatura. A experiência aqui relatada é importante para a construção de saberes na prática assistencial dos enfermeiros, além disso, este estudo favoreceu a identificação das necessidades de cuidados específicos para essa doença. Dentre as limitações desse estudo está no número de indivíduos estudados. Como já foi afirmado, trata-se de um relato de experiência, sendo pertinente que futuros trabalhos relacionados a assistência à saúde envolvam maior número de indivíduos com essa doença.

\section{REFERENCIAS}

1. Anguita M, Villanueva E, Castillo JC, Cejudo L, Aguilera J, Suárez de Lezo J. Manejo terapéutico del paciente con arteriopatía no coronaria (enfermedad arterial periférica y/o enfermedad cerebrovascular): más allá de los síntomas. Rev Esp Cardiol. Supl. 2009; 9:49-55. 
2. American Diabetes Association. Peripheral Arterial Disease in People With Diabetes. Diabetes Care. 2013; 26:12. 3. Garofolo L, Ferreira SRG, Miranda JF. Study of Risk Factors Associated with Peripheral Arteriopathy in JapaneseBrazilians from Bauru (SP). Arq. Bras. Cardiol.. 2014, vol.102, n.2.

4. Ministério da Saúde. Secretaria de Atenção à Saúde. Departamento de Atenção Básica. Estratégias para o cuidado da pessoa com doença crônica : diabetes mellitus / Ministério da Saúde, Secretaria de Atenção à Saúde, Departamento de Atenção Básica. - Brasília : Ministério da Saúde, 2013.

5. American Diabetes Association. Diagnosis and classification of diabetes mellitus. Diabetes Care 2011;34 Suppl 1:S62-9.

6. Lucas LPP, Barichello E, Zuffi FB, Barbosa MH. A percepção dos portadores de Diabetes Mellitus tipo 2 em relação à amputação. Rev. Eletr. Enf. 2010;12(3):535-8.

7. Sociedade Brasileira de Cardiologia. VI Diretrizes Brasileiras de Hipertensão. Arquivos Brasileiros de Cardiologia, São Paulo, v. 95, n. 1, p. 1-51, 2010.

8. Aragão JA, Reis FP, Neto RRB, Aragão MECS, Nunes MAP, Feitosa VLC. Prevalência da doença arterial obstrutiva periférica em doentes com insuficiência renal crônica. J. vasc. bras. 2009, vol.8, n.4, pp. 301-306.

9. BRASIL. Agência Nacional de Vigilância Sanitária. Saúde e economia. 2011; 6:4-1 [acesso 03 fev 2016]. Disponível em: http://portal.anvisa.gov.br/ 10. Pedrosa DFA, Pelegrin AKAP, Siqueira HBOM, Silva TCR, Colhado OCG, Sousa FAEF. Avaliação da qualidade de vida em clientes com dor crônica isquêmica. Rev. Latino-Am Enfermagem. 2011; 19(1).

11. Cavalcante BLL, Lima UTS. Relato de experiência de uma estudante de Enfermagem em um consultório especializado em tratamento de feridas. J
Nurs Health, Pelotas (RS) 2012 jan/jun;1(2):94-103.

12. Hermann AP, Lacerda MR. Atendimento domiciliar à saúde: um relato de experiência. Cogitare Enferm 2007 Out/Dez; 12(4):513-8.

13. Hausmann M, Peduzzi M. Articulação entre as dimensões gerencial e assistencial do processo de trabalho do enfermeiro. Texto Contexto Enferm, Florianópolis, 2009 Abr-Jun; 18(2): 258-65.

14. Diagnósticos de enfermagem da NANDA: definições e classificação 20122014/ [NANDA International]. Porto Alegre: Artmed, 2013, 606p.

15. Barros ALBL. Classificações de diagnóstico e intervenção de enfermagem: NANDA-NIC. Acta Paul Enferm 2009;22(Especial - 70 Anos):864-7.

16. Dochterman JM, Bulechek GM. Classificação das intervenções de enfermagem (NIC). 4a. ed. Porto Alegre: Artmed; 2008.

17. Carvalho FIC, Silva JPN, Bittencourt MC, Brito Junior LC. Uso de papaína no tratamento de lesões ulcerativas de pacientes portadores de pé diabético: relato de cinco casos. Revista Paraense de Medicina V.24 (2) abril-junho 2010.

Recebido em 22/02/2016

Aprovado em 03/10/2016

Publicado em 29/12/2016 